\title{
Differential expression of angiogenic and anti-angiogenic molecules in the chick embryo chorioallantoic membrane and selected organs during embryonic development
}

\author{
CHRISTIAN MARINACCIO, BEATRICE NICO and DOMENICO RIBATTI* \\ Department of Basic Medical Sciences, Neurosciences, and Sensory Organs, University of Bari Medical School, \\ Bari, Italy
}

\begin{abstract}
In this study we have investigated by real time polymerase chain reaction (RT-PCR) the expression of cytokines, which are well known angiogenic and anti-angionenic molecules, during chick embryo development in four organs, namely the chorioallantoic membrane (CAM), heart, liver and optic tectum at four different stages. We have studied the expression of vascular endothelial growth factor-A (VEGF-A), fibroblast growth factor-2 (FGF-2), angiopoietin-1 (ANG-1), hypoxia inducible factor-1 and -2 alpha (HIF-1 $\alpha$ and HIF-2 $\alpha$ ), hepatocyte growth factor (HGF), and of endostatin. All the pro-angiogenic cytokines examined showed a progressive increase in their expression in brain, heart, and liver, through all the stages of development, and parallel endostatin reduces its expression. In contrast, in the CAM, all the pro-angiogenic factors examined, with the exception of ANG-1, showed a stably-decreased expression during development, whereas endostatin progressively increased its expression. The CAM as an extraembryonic membrane which mediates gas and nutrient exchange until hatching, may be considered an outer organ and not an intrinsic organ of the developing embryo in which the mechanisms regulating the development of the vascular tree are different.
\end{abstract}

KEY WORDS: angiogenesis, chorioallantoic membrane, chick embryo, heart, liver, optic tectum

\section{Introduction}

There is an extensive literature of the genetic background, the molecular and cellular mechanisms responsible for blood vessel formation. During embryonic development, the vasculature is formed by both vasculogenesis and angiogenesis (Risau, 1995, Risau and Flamme, 1995). The nascent vasculature is stabilized via the recruitment of mural cells, namely pericytes and smooth muscle cells, and the generation of the extracellular matrix. Vasculogenesis seems to be restricted to early developmental periods, whereas angiogenesis can occur during the entire life span but also occurs in the early embryo. Although establishment of the vasculature of most organs occurs by angiogenesis, development of the vasculature of certain endodermal organs, including liver, lung, pancreas, stomach/intestine, and spleen, occurs by vasculogenesis (Pardanaud and Dieterlen-Lievre, 1999).

Angiogenesis is controlled by the balance between molecules that have positive and negative regulatory activity and this concept had led to the notion of the "angiogenic switch", which depends on an increased production of one or more positive regulators of angiogenesis (Ribatti et al., 2007). The control mechanisms involved in the regulation of angiogenesis might be better investigated during embryonic development, because the vascular pattern is reproducible and forms in the absence of inflammatory processes. Vascularendothelial growth factor (VEGF) is most likely the primary factor involved as both a diffusible attractant for migrating endothelial cells and as substrate-bound vessel stabilization factor once vascular tubes are formed. Fibroblast growth factor-2 (FGF-2) and VEGF play a crucial role in the angiogenic expansion of the early network. Angiopoietins (ANGs) play a critical role in endothelial sprouting, vessel wall remodeling and mural cell recruitment. Disruption of ANG-1 is responsible for a failure of endothelial cells to associate properly with pericytes during embryonic angiogenesis (Suri et al., 1996). Moreover, during embryonic development of

\footnotetext{
Abbreviations used in this paper: ANG-1, angiopoietin-1; CAM, chorioallantoic membrane; FGF-2, fibroblast growth factor-2; HGF, hepatocyte growth factor; HIF- $1 \alpha$ hypoxia inducible factor-1 alpha; VEGF-A, vascular endothelial growth factor-A.
}

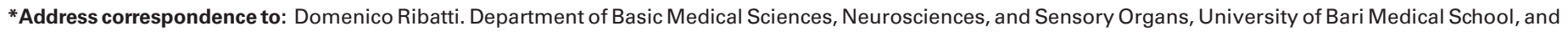

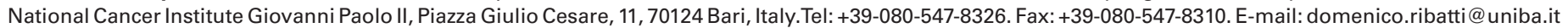




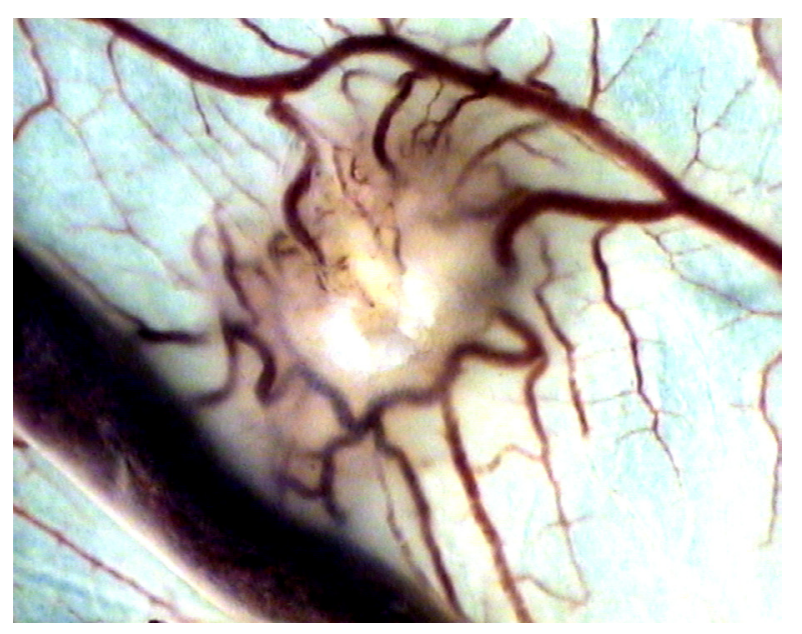

stages (E7, E12, E14, and E18), and we have compared the expression of angiogenic and anti-angiogenic molecules between heart, liver, optic tectum and CAM at different stages.

\section{Results}

\section{Relative expression of pro- and anti-angiogenic factors in the developing CAM}

All the pro-angiogenic factors examined, with the exception of ANG-1 and HIFs, showed a stably-decreased expression during development (Fig. 2). On the contrary, endostatin progressively increased its expression. VEGF-A, FGF-2 and HGF showed a peak of expression at ED7 of CAM development comparable to the reference expression at ED18, while ANG-1 and HIF-2 $\alpha$ display the peak of expression at ED12 after an initial lower expression at ED7. HIF-1 $\alpha$ showed a low expression at ED7

Fig. 1. A strong angiogenic response induced by a tumor bioptic specimen implanted on the top of a chick embryo chorioallantoic membrane at ED12. Original magnification, $x 50$.

the vascular system, hypoxia activates hypoxia inducible factor-1 and 2-alpha (HIF-1 $\alpha$ and HIF$2 \alpha$ ), which turn on the expression of angiogenic genes, such as VEGF, inducing vessels to branch towards the hypoxic tissue (Pugh and Ratcliffe, 2003). Among the anti-angiogenic molecules, endostatin is a proteolytic fragment of collagen XVIII (a component of basement membrane), which is able to inhibit endothelial cell survival and migration (Ribatti, 2009).

In this study we have investigated by Real time polymerase chain reaction (RT-PCR) the expression of well known angiogenic cytokines, VEGF-A, FGF-2, ANG-1, HIF- $1 \alpha$ and HIF-2 $\alpha$, hepatocyte growth factor (HGF) and of an endogenous antiangiogenic molecule, namely endostatin during chick embryo development. We have chosen four organs, namely chorioallantoic membrane (CAM), a useful model to study angiogenesis in ovo (Fig. 1 ), heart, liver and optic tectum at four different

Fig. 2. Relative expression of VEGF-A, FGF-2, ANG-1, HGF, HIF- $1 \alpha$, HIF-2 $\alpha$, and endostatin in the developing chorioallantoic membrane at ED7, 12, 14 of incubation in comparison to expression at E18 assumed as reference stage. All the pro-angiogenic factors except ANG-1 display a decreased expression during development, while endostatin shows an increased expression. A peak in expression can be seen at ED7 for VEGF-A, FGF-2 and HGF and at ED12 for ANG-1 and HIF-2 $\alpha$. HIF$1 \alpha$ shows a stable increase of expression at ED12 and ED14, while endostatin increased its expression at ED14 after a stable expression at ED7 and ED12. ${ }^{*} p<0.05 \mathrm{vs}$ $C A M$ at ED7. List of abbreviations: CAM, chorioallantoic membrane; VEGF-A, vascular endothelial growth factor-A; FGF-2, fibroblast growth factor-2; ANG-1, angiopoietin-1; $H G F$, hepatocyte growth factor; HIF-1 $\alpha$ and HIF-2 $\alpha$, hypoxia inducible factor 1 alpha and 2 alpha; ED, embryonic day.

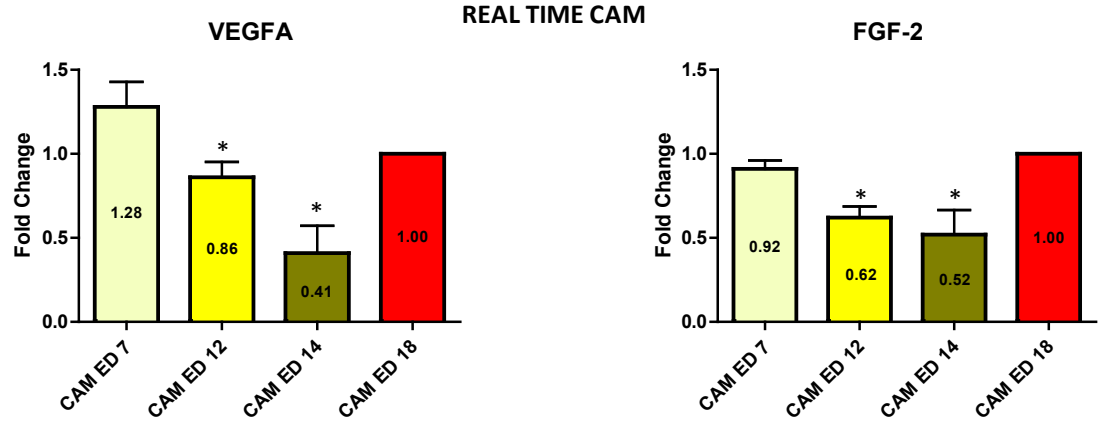

ANG-1
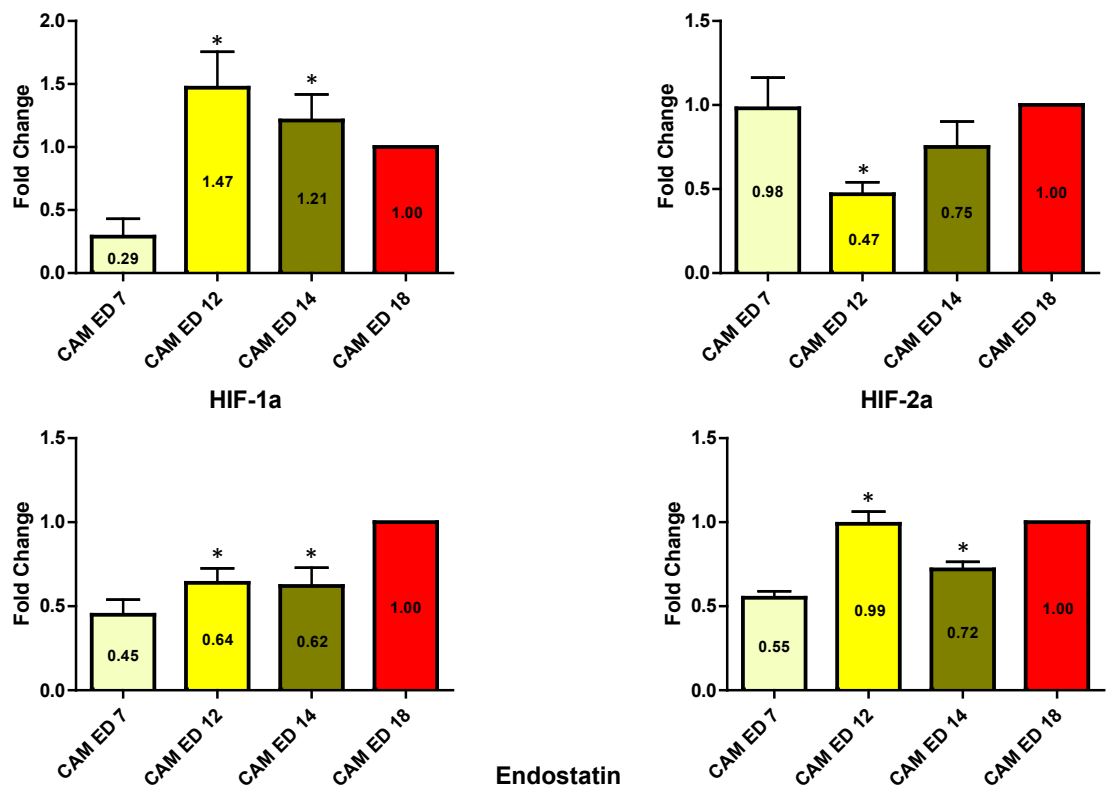

Endostatin

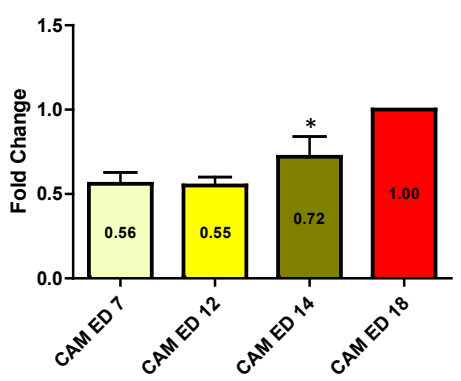


followed by a stable increase at ED12 and ED14. Endostatin increased its expression at ED14 after an initial stable expression at ED7 and ED12.

\section{Relative expression of pro- and anti-angiogenic factors in the developing heart}

All the factors analyzed showed a higher expression through the ED7, ED12, ED14 stage of heart development considered when compared to the ED18 CAM, with the exception of HIF-2 $\alpha$, which relative expression was substantially lower or comparable to the ED18 CAM (Fig. 3). After a stable expression in day 7 and
12, VEGF-A showed a peak in its expression during the 14 day stage and a subsequent decrease at day 18 of heart development. FGF-2 showed a stable expression through ED7, ED12 and ED14 and a decrease at ED18. ANG-1 showed a decreasing trend starting with a strong expression at ED7 followed by a reduction at ED12, ED14 and ED18 of heart development. Conversely, HGF showed a progressive increasing trend from ED7 through ED12 and ED14, followed by a strong decrease in its expression at ED18 of heart development. HIF-1 $\alpha$ showed a fluctuating expression from ED7 through ED12, ED14 and ED18 of heart development. After a stable expression in ED7 and ED12, HIF-2 $\alpha$

\section{REAL TIME PCR HEART-CAM}
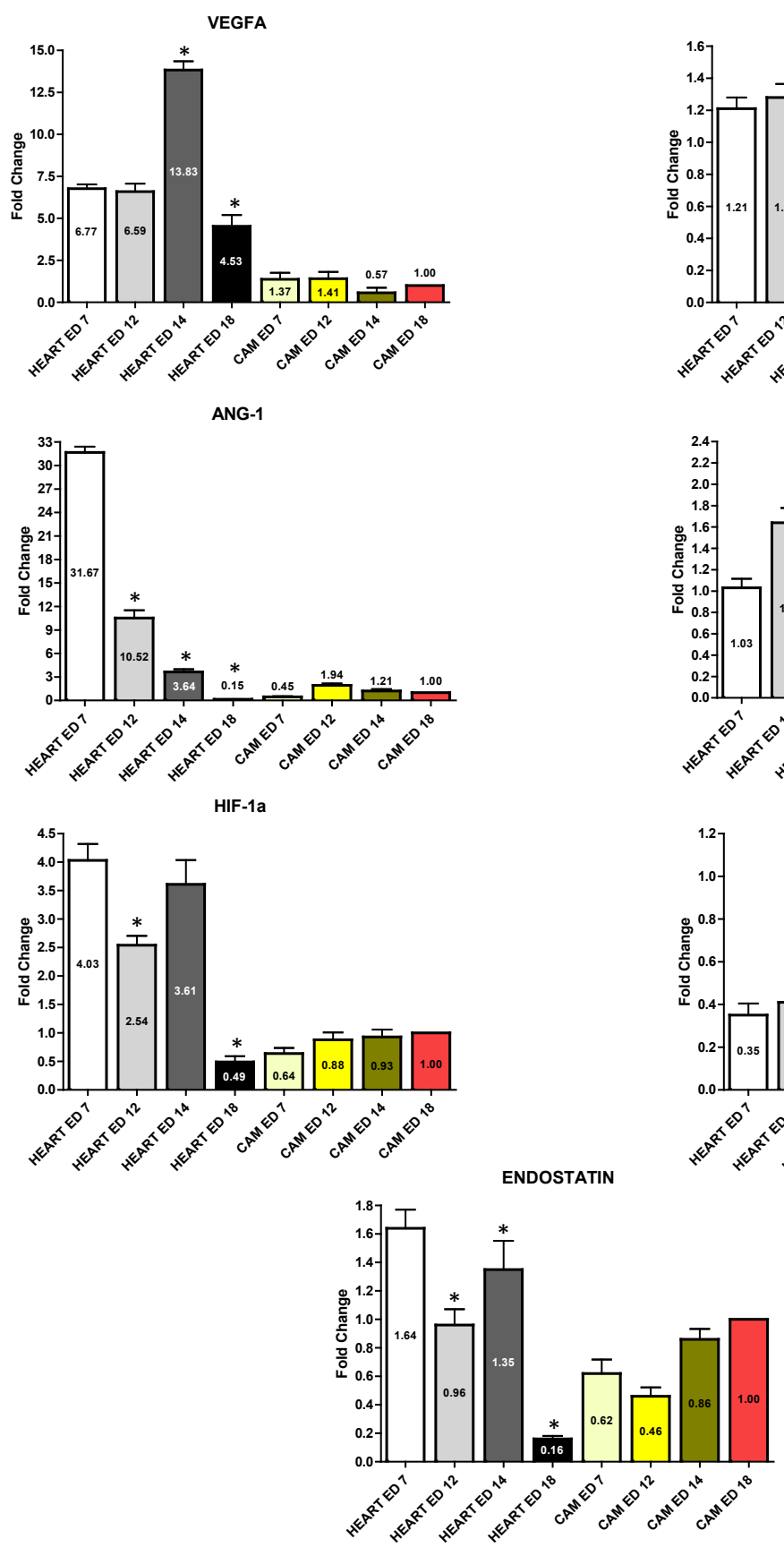

FGF-2

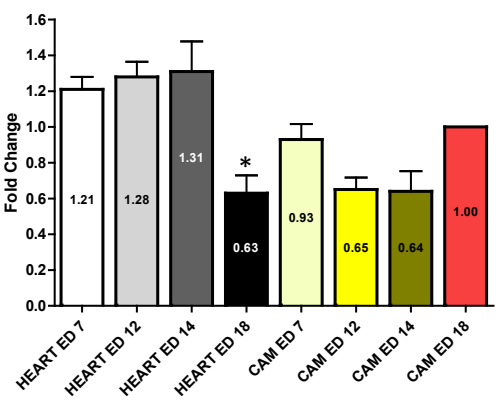

HGF
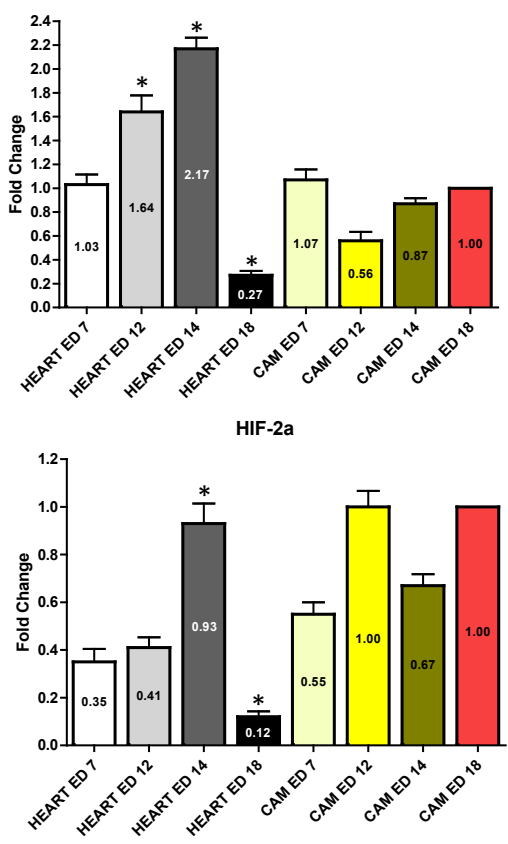

showed a peak in its expression during the ED14 and a subsequent decrease at ED18 of heart development. Endostatin showed a fluctuating expression from ED7 through ED12, ED14 and a reduction at ED18 of heart development. For all pro-angiogenic and anti-angiogenic factors examined, the lower expression was reached at ED18.

Percentage change of expression of pro- and anti-angiogenic factors between CAM and heart at different stages of development

All the factors considered, with the exception of HIF-2 $\alpha$, showed a positive percentage change of expression when compared to the CAM in all the stages of development, except for ED18, when FGF-2, ANG-1, HGF, HIF-2 $\alpha$ and endostatin showed a negative percentage change (Fig. 4). HIF-2 $\alpha$ showed an initial decrease at ED7 and ED12 followed by an increase at ED14. HGF showed the highest percentage increases at ED12, VEGF and FGF-2 at ED14, while ANG-1 and endostatin showed a progressive decrease after an initial high positive percentage change at ED7.

Relative expression of pro- and antiangiogenic factors in the developing liver

VEGF-A, FGF-2, HIF- $1 \alpha$ and HIF- $2 \alpha$ showed a peak in their relative expression at ED14 and a significant reduction at ED18 of liver development (Fig. 5).

Fig. 3. Relative expression of VEGF-A, FGF-2, ANG-1, HGF, HIF-1 $\alpha$, HIF-2 $\alpha$, and endostatin in the developing heart at ED7, 12,14 and 18 in comparison to CAM expression. All the factors except HIF-2 $\alpha$ display an higher expression during heart development than the expression during CAM development. A peak of expression was detected during ED14 of heart development in all the factors except for HIF-1 $\alpha$, ANG-1, Endostatin in which the peak is at ED7. ${ }^{*}<<0.05$ vs heart at ED7. List of abbreviations: CAM, chorioallantoic membrane; VEGF-A, vascular endothelial growth factor-A; FGF-2, fibroblast growth factor-2; ANG-1, angiopoietin-1; HGF, hepatocyte growth factor; HIF-1 $\alpha$ and HIF-2 $\alpha$, hypoxia inducible factor 1 alpha and 2 alpha; $E D$, embryonic day. 
HGF, a specific growth factor for the liver, showed a peak in its expression at the beginning of the chick embryo development suggesting an early role for this factor in the liver development. ANG-1 also showed a peak of its relative expression at ED7 of liver development. HIF- $1 \alpha$ and HIF-2 $\alpha$ showed a comparable trend through all the stages of development. Endostatin showed a significant reduction at the end of development. Percentage change of expression of pro- and anti-angiogenic factors between CAM and liver at different stages of development (Fig. 6)
VEGF-A was the only factor showing positive percentage changes during all stages of liver development with a peak at ED14. FGF-2, HIF-2 $\alpha$ and endostatin showed a negative percentage change of expression during all the stages of liver development. HIF- $1 \alpha$ showed the same trend with the exception of ED14 where the percentage change was positive, while HGF had its only negative percentage change at ED18. ANG-1 showed a peak of positive percentage change at ED7 and a negative percentage change from ED12 to ED18.
VEGF-A

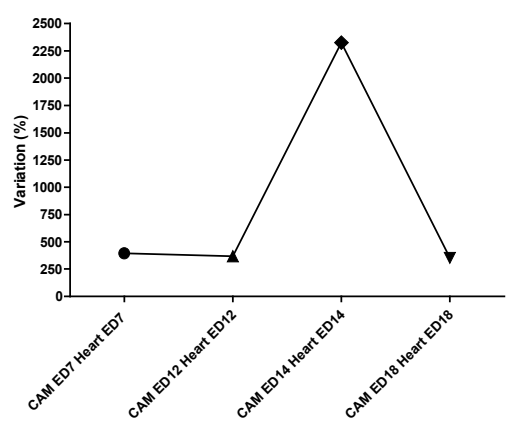

ANG-1
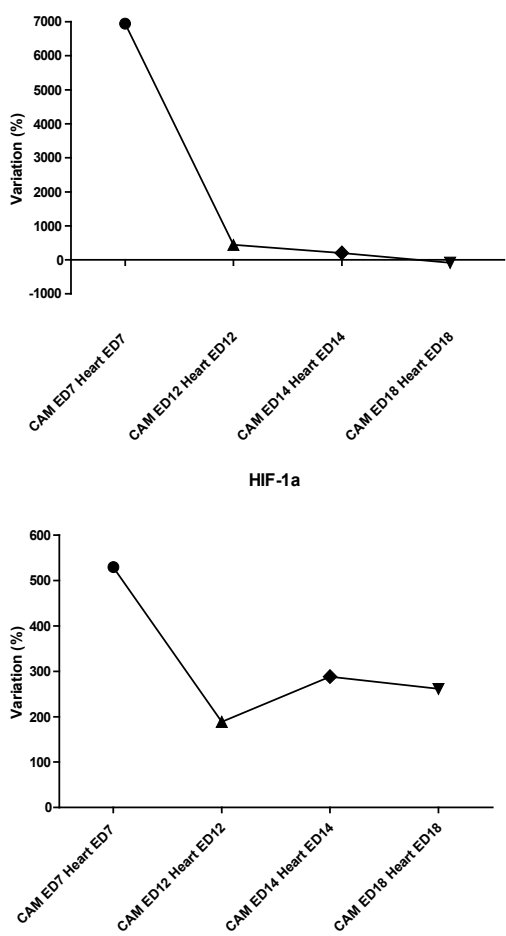

FGF-2

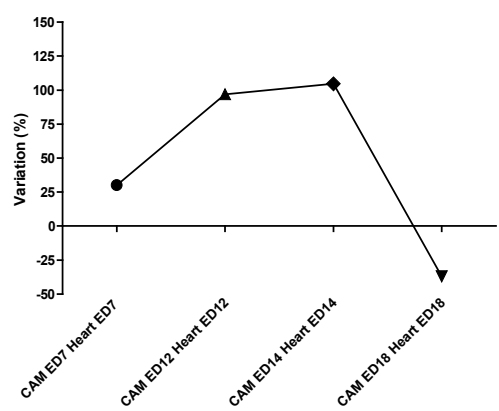

HGF
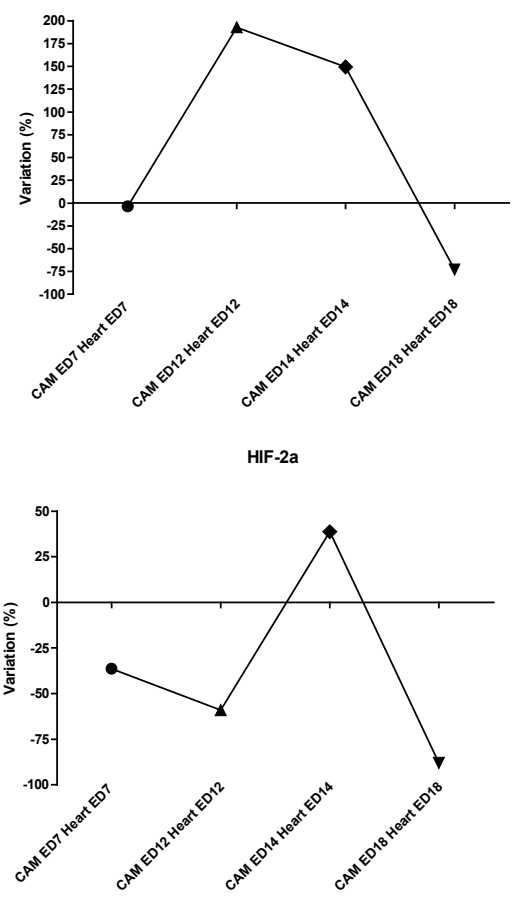

Relative expression of pro-and anti-angiogenic factors in the developing optic tectum

VEGF-A, ANG-1, HGF, HIF-1 $\alpha, \mathrm{HIF}-2 \alpha$ showed a progressive increase of relative expression through all the stages of the optic tectum development with a peak at ED18 (Fig. 7). FGF-2 showed a peak of relative expression at ED12 of optic tectum development. Endostatin reduced its expression at ED12 and slightly increased at ED14 and ED18.

\section{Percentage change of expression of pro- and anti-angiogenic factors between CAM and op- tic tectum at different stages of development}

VEGF-A, FGF-2 and HGF showed a progressive positive percentage change during ED7, ED12 and ED14 of the chick embryo development and a reduction at ED18 (Fig. 8). ANG-1 reached a positive percentage change peak towards the end of chick embryo development. HIF-1 $\alpha$ and HIF-2 $\alpha$ showed a positive and, respectively, a negative variation during all the stages of the chick embryo development. Endostatin showeda progressive decrease of percentage change from positive at ED7 to negative in the other stages of chick embryo development.

\section{Discussion}

The intricate patterning process that establish the complex architecture of the vascular system depend on a combination of genetic and epigenetic factors (Ribatti, 2006). It currently appears that blood vessels have the ability to provide instructive regulatory signals to surrounding non vascular target cells (Crivellato et al., 2007). On one hand, organ instructions to endothelial cells provides cues for vascular development and acquisition of local specialties; on the other hand, endothelial signals back to organ cells provide

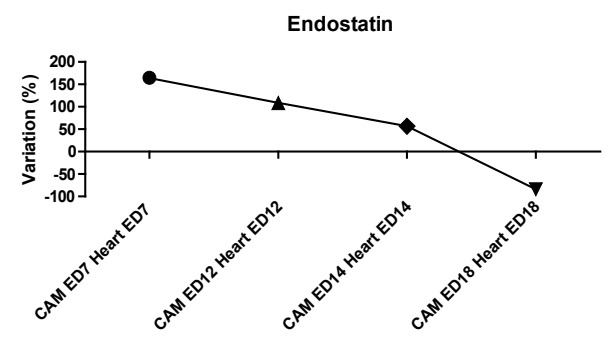

Fig. 4. Percentage change of expression of VEGF-A, FGF-2, ANG-1, HGF, HIF-1 $\alpha$, HIF-2 $\alpha$, and endostatin between CAM and heart at ED7, 12, 14 and 18. A positive percentage change can be seen in all the factors for ED7, ED12 and ED14 except HIF-2 $\alpha$. At ED18 FGF-2, ANG-1, HGF, HIF-2 $\alpha$ and endostatin display a negative percentage change. List of abbreviations: CAM, chorioallantoic membrane; VEGF-A, vascular endothelial growth factor-A; FGF-2, fibroblast growth factor-2; ANG-1, angiopoietin-1; HGF, hepatocyte growth factor; HIF-1 $\alpha$ and HIF-2 $\alpha$, hypoxia inducible factor 1 alpha and 2 alpha; ED, embryonic day. 
morphogenetic and patterning instructions during organ formation (Cleaver and Melton, 2003).

In this study, we have demonstrated that all the pro-angiogenic cytokines examined show a progressive increase in their expression in brain, heart, and liver, through all the stages development, and parallel endostatin reduces its expression. On the contrary in the $\mathrm{CAM}$, all the pro-angiogenic factors examined, with the exception of ANG-1, showed a stably decreased expression during development, whereas endostatin progressively increased its expression.
Few literature data are available concerning the time-course in the expression of angiogenic and anti-angiogenic molecules during the development of different organs in the chick embryo. As concerns the CAM, primitive vessels proliferate and differentiate into an arteriovenous system until day 8 , thus originating a network of capillaries that migrate to occupy an area beneath the chorion and mediate gas exchanges with the outer environment. Rapid capillary proliferation goes until ED11; thereafter, their mitotic index declines just as rapidly, and the vascular system attains its

final arrangement on ED18, just before hatching (Ausprunk et al., 1974). A morphometric investigation by DeFouw et al., (1989) has shown rapid extension of the CAM surface between ED6 and ED14. During this period, the number of feed vessels increased predominantly due to growth and remodeling after ED10. Dusseau and Hutchins (1989) studied the effects of hypoxia on the number of pre and post-capillary vessels in the CAM and demonstrated that the low oxygen regimen stimulated a preferential increase in the number of arterioles. Ribatti et al., (1995) demonstrated that endogenous FGF-2 is intrinsically involved in CAM vascularization on the basis of the evidence that FGF-2 is present in elevated amounts in the CAM from ED6 to ED18, maximal concentrations being observed between ED10 and ED14. We have confirmed the highest amount of FGF-2 between ED7 and ED12. Moreover, neutralizing antibodies to FGF-2 inhibit vessel growth, confirming a role of FGF-2 in vascularization of the CAM. Parson-Wingerter et al., (1998) demonstrated that FGF-2 increased the rate of angiogenesis in the CAM by a maximum of $72 \%$, whereas angiostatin decreases the rate of vascular growth by a maximum of $68 \%$.

As concerns the central ner-
ENDOSTATIN

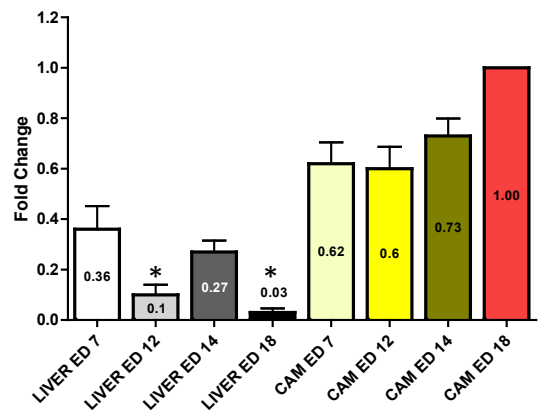

Fig. 5. Relative expression of VEGF-A, FGF-2, ANG-1, HGF, HIF-1 $\alpha$ HIF-2 $\alpha$, and endostatin in the developing liver at ED7, 12, 14 and 18 in comparison to CAM expression. A peak of expression of VEGF-A, FGF-2, HIF-1 $\alpha$ and HIF-2 $\alpha$ can be noted at ED 14 followed by a significant reduction at ED18 of liver development. ANG-1 and HGF showed a peak at ED7 of liver development while endostatin showed a significant reduction at ED18. ${ }^{*} p<0.05$ vs liver at ED7. List of abbreviations: CAM, chorioallantoic membrane; VEGF-A, vascular endothelial growth factor-A; FGF-2, fibroblast growth factor-2; ANG-1, angiopoietin-1; HGF, hepatocyte growth factor; HIF-1 $\alpha$ and HIF-2 $\alpha$, hypoxia inducible factor 1 alpha and 2 alpha; ED, embryonic day. 

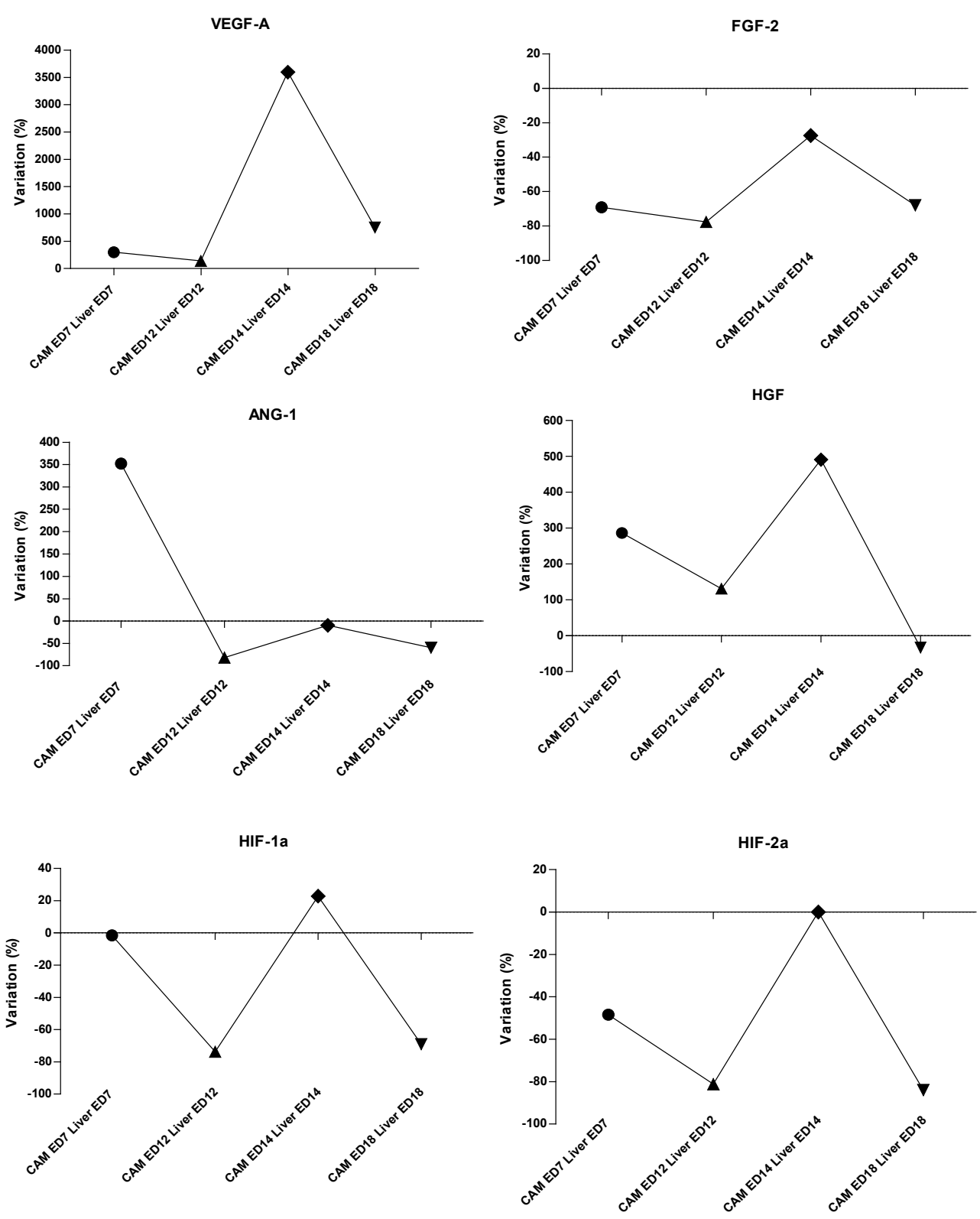

Endostatin

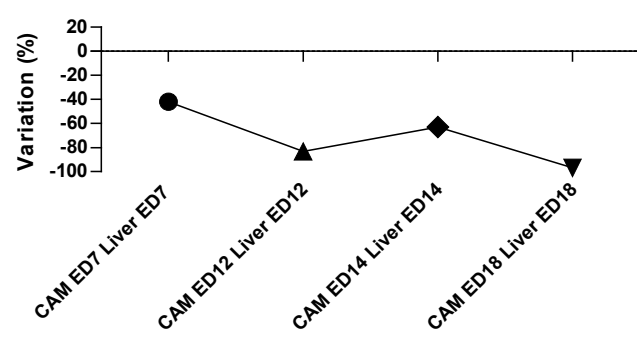

Fig. 6. Percentage change of expression of VEGF-A, FGF-2,ANG-1, HGF, HIF-1 $\alpha$, HIF-2 $\alpha$, and endostatin between CAM and liver at ED7, 12, 14 and 18. Only VEGF-A displays a positive percentage change during all liver development, while FGF-2, HIF-2 $\alpha$ and endostatin display an opposite trend. ANG-1, HGF and HIF-1 $\alpha$ showed a peak of positive percentage change at ED7 and ED14 respectively. List of abbreviations: CAM, chorioallantoic membrane; VEGF-A, vascular endothelial growth factor-A; FGF-2, fibroblast growth factor-2; ANG-1, angiopoietin-1; HGF, hepatocyte growth factor; HIF-1 $\alpha$ and HIF-2 $\alpha$, hypoxia inducible factor 1 alpha and 2 alpha; ED, embryonic day. vous system, angioblasts invade the head and form a perineural vascular plexus that covers the neural tube. Vascular sprouts originating from this plexus radially invade the neuroectoderm (Bar, 1980). Subsequently, branching occurs in the subependymal layer. Vascular endothelial cells are among the first cells encountered by neuroblasts during early development. Neuroblasts release endothelial cell mitogens, which promote angiogenesis by the invading vasculature and endothelial cells release factors that support neuronal development (Bautch and James, 2009).

In normally developing chick embryo optic tectum, the blood vessels enter the nervous wall as radial vessels at ED 5-6, and from ED8, the number of these main vessels and of their collateral increases owing to progressively more numerous branching vessels, parallel to the main tectum histogenetic processes (Roncali et al., 1985). In hypoxic condition, the number of vessels was increased; a transitory increment in vessel diameter was recognized and the value of the distance between contiguous vessels was much lower than under normal condition (Ribatti et al., 1989). Risau isolated from embryonic chick brain an endothelial cell growth factor and found to be identical to acidic and basic fibroblast growth factor (Risau, 1986, Risau et al., 1988). The mitogenic activity was found to increase during embryonic chick brain development and reached a plateau around ED14. In this study, we have confirmed the highest amount of FGF-2 between ED12 and ED18. Breier et al., (1992) demonstrated that the temporal and spatial expression of VEGF in the developing brain correlates with brain vascularization and endothelial cell growth. They suggested that VEGF is releases by cell of the ventricular layer and promotes angiogenesis by initiating an angiogenic response of endothelial cells from the perineural vascular plexus. In this study, we have demonstrated an high expression of VEGF-A between ED12 and ED18.

The heart develops form the precardic lateral folds to form primitive heart tube, consisting of an inner endothelium and an outer myocardial 
tube. Emergence of cardiac endothelium and cardiomyocytes occurs almost concomitantly and, at first, they develop rather independently from one another (Sugi and Markwald, 1996).

Experimental data in mouse and zebrafish support the view that endocardial cells play a critical role in myocardial cell maturation. A study concerning immunolocalization of FGF-2 during chicken cardiac development demonstrated a progressive decrease in its expression in the highly trabeculated region of the ventricular myocardium with increasing developmental stage (Consigli and Joseph-Silverstein, 1991).

Tomanek et al., (1998) tested the hypothesis that early vascularization of the embryonic heart prior to myocardial tube formation is enhanced after bolus injection of VEGF and FGF-2 into the vitelline vein before the onset of myocardial vascularization and demonstrated increased vascular growth. Guadix et al., (2006) demonstrated that chick epicardium displays a high vasculogenic potential which is modulated by VEGF and FGF-2. Tomanek et al., (2002) have documented a role for multiple members of the VEGF family and their receptors in the formation of the coronary vascular bed in quail heart. Sugishita et al., (2000) suggested that VEGF produced by chick embryonic heart myocytes may play an important role in embryonic cardiovascular development by acting on surrounding endothelial cells and on ventricular myocytes themselves. Tomanek et al., (2001) demonstrated that addition to heart explants of anti-VEGF antibodies inhibited vascular tube formation by about $25 \%$, while anti-FGF-2 antibodies affected a $60 \%$ inhibition; when a combination of two inhibitors was added, tubulogenesis was inhibited by $80-90 \%$. In this study, we have confirmed an important role played by VEGF-A and FGF-2 during heart development.

Song et al., (1999) using an immunohistochemical approach detected a localization of HGF to the chick myocardial cells and demonstrated that isolated endocardial cells responded to the addition of HGF with increased motility, proliferation and urokinase production. Wikenheiser et al., (2006) identified areas of the embryonic chicken heart that were intensely positive for the hypoxia indicator (EF5) and these areas were also positive for HIF$1 \alpha$. In this study, we have demonstrated in heart samples a strong expression of both HGF and HIF-1 $\alpha$ at ED14.

The liver anlagen develops from the ventral foregut endothelium (Wells and Melton, 1999). Endodermal epithelial cells of the primitive intestine receive stimuli from the cardiac mesoderm which induces changes in endodermal gene profile with expression of liver specific genes (Jung et al., 1999). As a consequence, endodermal cells differentiate and proliferate within the foregut epithelium, and begin to delaminate into the surrounding mesenchyme of the septum transversum. Finally, the condensed tissue mass becomes vascularized (Matsumoto et al., 2001).

HGF is a cytokine that stimulates pro-
ENDOSTATIN

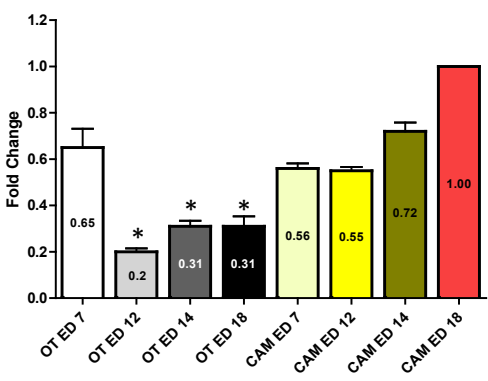

Fig. 7. Relative expression of VEGF-A, FGF-2, ANG-1, HGF, HIF- $1 \alpha$, HIF-2 $\alpha$, and endostatin in the developing optic tecum at ED7, 12, 14 and 18 in comparison to CAM expression. A progressive increase of expression in optic tectum development can be seen for VEGF-A, ANG-1, HGF, HIF-1 $\alpha$ and HIF-2 $\alpha$ with a peak at ED18, while a progressive reduction of expression of endostatin is present, with the lowest expression at ED12. FGF-2 displays a peak of expression at ED12 of optic tectum development. ${ }^{*} p<0.05$ vs optic tectum at ED7. List of abbreviations: CAM, chorioallantoic membrane; VEGF-A, vascular endothelial growth factor-A; FGF-2, fibroblast growth factor-2; ANG-1, angiopoietin-1; HGF, hepatocyte growth factor; HIF-1 $\alpha$ and HIF-2 $\alpha$, hypoxia inducible factor 1 alpha and 2 alpha; ED, embryonic day. 

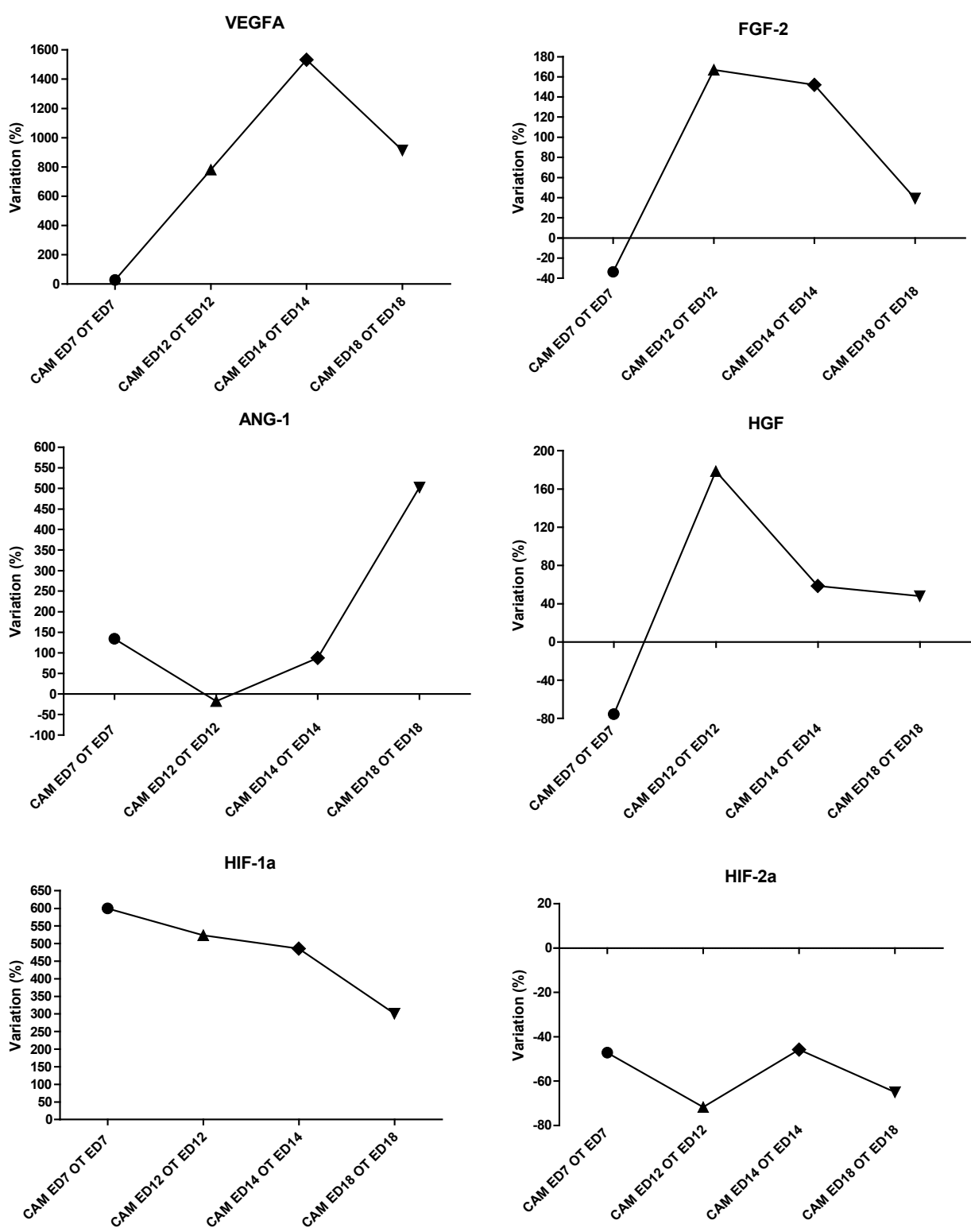

liferation and morphogenesis of epithelia and is a hepatrophic factor for liver repair (Nakamura et al., 1989). Moreover, HGF is a potent angiogenic molecule through direct actions on endothelial cells, including stimulation of cell motility, proliferation, protease production, invasion, and organization into capillary-like tubes (Bussolino et al., 1992). Endothelial cells are essential for early and later stages of liver development. Accordingly, sinusoid endothelial cells supply HGF, which promotes hepatocyte growth and expansion of the liver mass (LeCouter et al., 2003). We have confirmed an important role played by HGF during chick liver organogenesis, due to its highest expression in the first stage of development examined at ED7.

Overall, in this study we have compared for the first time the expression of well known angiogenic cytokines and of one endogenous inhibitor of angiogenesis in four different organs of chick embryo in different developmental stages. We have demonstrated a common pathway of expression in liver, heart and optic tectum samples, showing a progressive increase in the expression of angiogenic cytokines and a parallel decrease in the expression of endostatin, whereas in the CAM this pathway is opposite. Probably, the CAM acting as an extraembryonic membrane mediating gas and nutrient exchanges until hatching (Romanoff, 1960), may be considered an outer organ and not an intrinsic organ of the developing embryo in which the mechanisms regulating the development of the vascular tree are different.

\section{Materials and Methods}

\section{Endostatin}

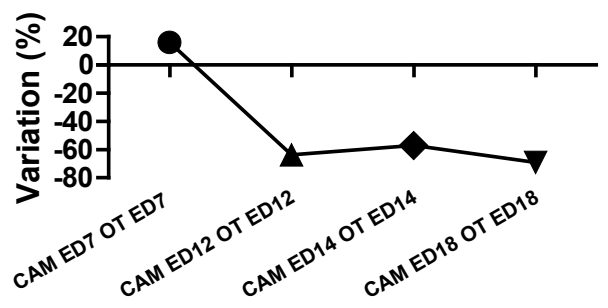

Fig. 8. Percentage change of expression of VEGF-A, FGF-2, ANG-1, HGF, HIF-1 $\alpha$, HIF-2 $\alpha$, and endostatin between CAM and optic tectum at ED7, 12, 14 and 18. A progressive positive percentage change is displayed by VEGF-A, FGF-2 and HGF with a reduction at ED18. HIF-1 $\alpha$ and HIF-2 $\alpha$ showed different percentage change trend with a positive and a negative variation respectively in the embryonic development. ANG-1 reached a positive percentage peak at the end of embryonic development while endostatin showed a decrease from positive at ED7 to negative at ED12, ED14 and ED18. List of abbreviations: CAM, chorioallantoic membrane; VEGF-A, vascular endothelial growth factor-A; FGF-2, fibroblast growth factor-2; ANG-1, angiopoietin-1; HGF, hepatocyte growth factor; HIF-1 $\alpha$ and HIF-2 $\alpha$, hypoxia inducible factor 1 alpha and 2 alpha; ED, embryonic day.

\section{Chorioallantoic membrane, heart, liver and optic tectum tissue sampling}

Two hundred fertilized White Leghorn chicken eggs were incubated at $37^{\circ} \mathrm{C}$ at constant humidity $(60 \%)$. On day 3 of incubation a square window was opened in the egg shell after removal of 2-3 $\mathrm{ml}$ of albumen to detach the developing CAM from the shell. The window was sealed with glass and the eggs were returned to the incubator. Embryo manipulation did not alter its development. The possibility to look inside the embryo through the window allows to verify the vitality of the embryo. Ten chick at embryonic day (ED) 7, 12, 14 and 18 of development were sacrificed. Ten hearts, 10 livers, 10 optic tecta and 10 CAM specimens from each chick embryo at each above mentioned stages were isolated and immersed in RNA Later Solution (Ambion, Austin, TX, 
TABLE 1

\section{SPECIFIC PRIMERS FOR CHICKEN TRANSCRIPTS FOR REAL-TIME PCR}

\begin{tabular}{|c|c|}
\hline Factor & Primer sequence (5'- 3') \\
\hline VEGF-A & $\begin{array}{l}\text { 5'-TCAGAGTCAGCACATAGC-3' } \\
\text { 5'-CCTTTCCTCGCTTTGATTT-3' }\end{array}$ \\
\hline FGF-2 & $\begin{array}{l}\text { 5'-GGCTATGAAGGAGGATGG-3' } \\
\text { 5'-TGCCACATACCAATCAGA-3' }\end{array}$ \\
\hline ANG-1 & $\begin{array}{l}\text { 5'-CAGTGGGAGAAGATTTAACC-3' } \\
\text { 5'-CTGAAGAGCGTTAGTGTTG-3' }\end{array}$ \\
\hline HIF- $1 \alpha$ & $\begin{array}{l}\text { 5'-CTTCAGCAGACTCAGACA-3' } \\
\text { 5'-ACAGGAGATGATGGAACAA-3' }\end{array}$ \\
\hline HIF- $2 \alpha$ & $\begin{array}{l}\text { 5'-CAGCAGAGTCTAAGTAGCA-3' } \\
\text { 5'-GTTGAAGAACACCGATGATAG-3' }\end{array}$ \\
\hline HGF & $\begin{array}{l}\text { 5'-TTCAGAGAACCAGATGTTAGTA-3' } \\
\text { 5'-GGAGTTGTGTCACCAGTA-3' }\end{array}$ \\
\hline Endostatin & $\begin{array}{l}\text { 5'-ATGAAGTCATCCTCTACCTG-3' } \\
\text { 5'-GAGCCTTCTTCTAGTTCCA-3' }\end{array}$ \\
\hline$\beta$-Actin & $\begin{array}{l}\text { 5'-CTTCCAGCCATCTTTCTT-3' } \\
\text { 5'-ATATCCACATCACACTTCAT-3' }\end{array}$ \\
\hline
\end{tabular}

USA) for subsequent RNA extraction. The experiments were performed in accordance with the international directions for the protection of animals used for scientific purposes and approved by the local ethical committee.

\section{RNA extraction and Real-time RT-PCR}

Total RNA was extracted from heart, liver and CAM samples using RNeasy mini kit (Quiagen, Hilden, Germany), while the RNeasy Lipid mini kit (Quiagen, Hilden, Germany) was used to extract total RNA from optic tectum samples. Total RNA was then reverse-transcribed into cDNAs using iScript cDNA Synthesis kit (Bio-Rad Laboratories, Hercules, CA, USA) according to the manifacturer's instructions. The cDNAs were used to amplify a panel of six pro-angiogenic and one anti-angiogenic factors with the $i Q$ SYBR Green Supermix (Bio-Rad Laboratories, Hercules, CA, USA). PCR amplification and Real-Time detection were performed using the Chromo4 Real-Time PCR Detection System (Bio-Rad Laboratories). Samples were normalized to chick beta-actin. Table 1 shows the sequences of chicken primers (Invitrogen) for each factor analyzed. Primers were designed using the software Beacon Designer 8 (PREMIER Biosoft International, Palo Alto, CA, USA).

All the experiments were performed with $60^{\circ} \mathrm{C}$ as the annealing temperature. The amplification process included 35 cycles, each consisting of three steps: incubation at $95^{\circ} \mathrm{C}$ for 3 minutes; incubation at $95^{\circ} \mathrm{C}$ for $15 \mathrm{~s}$; annealing and extension at $60^{\circ} \mathrm{C}$ for $30 \mathrm{~s}$. The melting curve analysis was performed at $55-95^{\circ} \mathrm{C}$ interval with $0.5^{\circ} \mathrm{C}$ temperature increases per reading step.

\section{Real time RT-PCR relative expression analysis}

The relative expression (fold change) of the pro- and anti-angiogenic factors in each stage of the chick embryo development was determined with the comparative Cycle threshold method $\left(2^{-\Delta \Delta C t}\right)$. Ct values from RT-PCR were normalized with the housekeeper gene $\beta$-actin and then compared with the latest stage considered of the CAM development at ED18. CAM of chick embryo at ED18 was chosen as the reference stage for expression analysis to establish a comparison between its angiogenic gene expression pattern and the one of other organs examined. Resulting Fold Change was plotted using GraphPad Prism 5 (GraphPad Software, Inc, San Diego, CA, USA).

\section{Percentage change data analysis}

Relative expression of heart, liver and optic tectum from each stage of development were compared with relative expression of CAM at the same stage of development of the above mentioned organs as percentage increase or decrease from CAM expression. Resulting percentage changes were plotted using GraphPad Prism 5 (GraphPad Software, Inc, San Diego, CA, USA).

\section{Statistical analysis}

Fold change data are reported as means \pm SD. Newman-Keuls multiple comparison post-test was used to compare all developmental stages following one-way ANOVA. The Graph Pad Prim 5.0 statistical package (GraphPad Software, San Diego, CA, USA) was used for the analysis and $\mathrm{P}<0.05$ was considered as the limit for statistical significance.

\section{Acknowledgements}

The research leading to these results received funding from the European Union Seventh Framework Programme (FP7/2007-2013) under grant agreement $n^{\circ} 278570$ to $D R$.

\section{References}

AUSPRUNK, D.H., KNIGHTON, D.R. and FOLKMAN, J. (1974). Differentiation of vascular endothelium in the chick chorioallantois: a structural and autoradiographic study. Dev Biol 38: 237-248

BAR, T. (1980). The vascular system of the cerebral cortex. Adv Anat Embryol Cell Biol 59: I-VI,1-62.

BAUTCH, V.L. and JAMES, J.M. (2009). Neurovascular development: The beginning of a beautiful friendship. Cell Adh Migr 3: 199-204.

BREIER, G., ALBRECHT, U., STERRER, S. and RISAU, W. (1992). Expression of vascular endothelial growth factor during embryonic angiogenesis and endothelial cell differentiation. Development 114: 521-532.

BUSSOLINO, F., DI RENZO, M.F., ZICHE, M., BOCCHIETTO, E., OLIVERO, M., NALDINI, L., GAUDINO, G., TAMAGNONE, L., COFFER, A. and COMOGLIO, P.M. (1992). Hepatocyte growth factor is a potent angiogenic factor which stimulates endothelial cell motility and growth. J Cell Biol 119: 629-641.

CLEAVER, O. and MELTON, D.A. (2003). Endothelial signaling during development Nat Med 9: 661-668.

CONSIGLI, S.A. and JOSEPH-SILVERSTEIN, J. (1991). Immunolocalization of basic fibroblast growth factor during chicken cardiac development. J Cell Physiol 146: 379-385.

CRIVELLATO, E., NICO, B. and RIBATTI, D. (2007). Contribution of endothelial cells to organogenesis: a modern reappraisal of an old Aristotelian concept. $J$ Anat 211: 415-427.

DEFOUW, D.O., RIZZO, V.J., STEINFELD, R. and FEINBERG, R.N. (1989). Mapping of the microcirculation in the chick chorioallantoic membrane during norma angiogenesis. Microvasc Res 38: 136-147.

DUSSEAU, J.W. and HUTCHINS, P.M. (1989). Microvascular responses to chronic hypoxia by the chick chorioallantoic membrane: a morphometric analysis. $\mathrm{Mi}$ crovasc Res 37: 138-147.

GUADIX, J.A., CARMONA, R., MUNOZ-CHAPULI, R. and PEREZ-POMARES, J.M. (2006). In vivo and in vitro analysis of the vasculogenic potential of avian proepicardial and epicardial cells. Dev Dyn 235: 1014-1026.

JUNG, J., ZHENG, M., GOLDFARB, M. and ZARET, K.S. (1999). Initiation of mammalian liver development from endoderm by fibroblast growth factors. Science 284: 1998-2003.

LECOUTER, J., MORITZ, D.R., LI, B., PHILLIPS, G.L., LIANG, X.H., GERBER, H.P., HILLAN, K.J. and FERRARA, N. (2003). Angiogenesis-independent endothelial protection of liver: role of VEGFR-1. Science 299: 890-893.

ATSUMOTO, K., YOSHITOMI, H., ROSSANT, J. and ZARET, K.S. (2001). Liver organogenesis promoted by endothelial cells prior to vascular function. Science 294: 559-563.

NAKAMURA, T., NISHIZAWA, T., HAGIYA, M., SEKI, T., SHIMONISHI, M., SUGIMURA A., TASHIRO, K. and SHIMIZU, S. (1989). Molecular cloning and expression of human hepatocyte growth factor. Nature 342: 440-443.

PARDANAUD, L. and DIETERLEN-LIEVRE, F. (1999). Manipulation of the angiopoietic/ hemangiopoietic commitment in the avian embryo. Development 126: 617-627.

PARSONS-WINGERTER, P., LWAI, B., YANG, M.C., ELLIOTT, K.E., MILANINIA, A., REDLITZ, A., CLARK, J.I. and SAGE, E.H. (1998). A novel assay of angiogenesis in the quail chorioallantoic membrane: stimulation by bFGF and inhibition by angiostatin according to fractal dimension and grid intersection. Microvasc 


\section{C. Marinaccio et al.}

Res 55: 201-214.

PUGH, C.W. and RATCLIFFE, P.J. (2003). Regulation of angiogenesis by hypoxia: role of the HIF system. Nat Med 9: 677-684.

RIBATTI, D. (2006). Genetic and epigenetic mechanisms in the early development of the vascular system. J Anat 208: 139-152.

RIBATTI, D. (2009). Endogenous inhibitors of angiogenesis: a historical review. Leuk Res 33: 638-644.

RIBATTI, D., MANCINI, L., RONCALI, L., NICO, B. and BERTOSSI, M. (1989). Morphometric analysis on the effects of hypoxia during the central nervous system vasculogenesis. Int J Microcirc Clin Exp 8: 135-142.

RIBATTI, D., NICO, B., CRIVELLATO, E., ROCCARO, A.M. and VACCA, A. (2007). The history of the angiogenic switch concept. Leukemia 21: 44-52.

RIBATTI, D., URBINATI, C., NICO, B., RUSNATI, M., RONCALI, L. and PRESTA, M. (1995). Endogenous basic fibroblast growth factor is implicated in the vascularization of the chick embryo chorioallantoic membrane. Dev Biol 170: 39-49.

RISAU, W. (1986). Developing brain produces an angiogenesis factor. Proc Natl Acad Sci USA 83: 3855-3859.

RISAU, W. (1995). Differentiation of endothelium. FASEB J 9: 926-933.

RISAU, W. and FLAMME, I. (1995). Vasculogenesis. Annu Rev Cell Dev Biol 11: 73-91.

RISAU, W., GAUTSCHI-SOVA, P. and BOHLEN, P. (1988). Endothelial cell growth factors in embryonic and adult chick brain are related to human acidic fibroblast growth factor. EMBO J 7: 959-962.

ROMANOFF, A.L. (1960). The extraembryonic membranes. In The Avian Embryo Structural and Functional Development. Mac Milian, New York, pp.1039-1141.

RONCALI, L., RIBATTI, D. and AMBROSI, G. (1985). Vasculogenesis in the chick embryo optic tectum. Acta Anat (Basel) 122: 229-234.

SONG, W., MAJKA, S.M. and MCGUIRE, P.G. (1999). Hepatocyte growth factor expression in the developing myocardium: evidence for a role in the regulation of the mesenchymal cell phenotype and urokinase expression. Dev Dyn214:92-100.

SUGI, Y. and MARKWALD, R.R. (1996). Formation and early morphogenesis of endocardial endothelial precursor cells and the role of endoderm. Dev Biol 175: 66-83.

SUGISHITA, Y., TAKAHASHI, T., SHIMIZU, T., YAO, A., KINUGAWA, K., SUGISHITA K., HARADA, K., MATSUI, H. and NAGAI, R. (2000). Expression of genes encoding vascular endothelial growth factor and its Flk-1 receptor in the chick embryonic heart. J Mol Cell Cardiol 32: 1039-1051.

SURI, C., JONES, P.F., PATAN, S., BARTUNKOVA, S., MAISONPIERRE, P.C., DAVIS S., SATO, T.N. and YANCOPOULOS, G.D. (1996). Requisite role of angiopoietin-1, a ligand for the TIE2 receptor, during embryonic angiogenesis. Cell87: 1171-1180.

TOMANEK, R.J., HOLIFIELD, J.S., REITER, R.S., SANDRA, A. and LIN, J.J. (2002) Role of VEGF family members and receptors in coronary vessel formation. Dev Dyn 225: 233-240.

TOMANEK, R.J., LOTUN, K., CLARK, E.B., SUVARNA, P.R. and HU, N. (1998). VEGF and bFGF stimulate myocardial vascularization in embryonic chick. $A m \mathrm{~J}$ Physiol 274: H1620-H1626.

TOMANEK, R.J., ZHENG, W., PETERS, K.G., LIN, P., HOLIFIELD, J.S. and SUVARNA P.R. (2001). Multiple growth factors regulate coronary embryonic vasculogenesis. Dev Dyn 221: 265-273.

WELLS, J.M. and MELTON, D.A. (1999). Vertebrate endoderm development. Annu Rev Cell Dev Biol 15: 393-410.

WIKENHEISER, J., DOUGHMAN, Y.Q., FISHER, S.A. and WATANABE, M. (2006). Differential levels of tissue hypoxia in the developing chicken heart. Dev Dyn 235: 115-123. 


\section{Further Related Reading, published previously in the Int. J. Dev. Biol.}

Zebrafish enhancer trap line recapitulates embryonic aquaporin 1a expression pattern in vascular endothelial cells Kira Rehn, Kuan Shen Wong, Darius Balciunas and Saulius Sumanas Int. J. Dev. Biol. (2011) 55: 613-618 http://dx.doi.org/10.1387/ijdb.103249kp

Zebrafish embryo, a tool to study tumor angiogenesis Chiara Tobia, Giulia De Sena and Marco Presta Int. J. Dev. Biol. (2011) 55: 505-509 http://dx.doi.org/10.1387/ijdb.103238ct

Anti-angiogenesis in cancer; met and unmet goals - an interview with Robert Kerbel Francesco Bertolini

Int. J. Dev. Biol. (2011) 55: 395-398

http://dx.doi.org/10.1387/ijdb.103217fb

A brief history of angiogenesis assays

Anca-Maria Cimpean, Domenico Ribatti and Marius Raica

Int. J. Dev. Biol. (2011) 55: 377-382

http://dx.doi.org/10.1387/ijdb.103215ac

ADAM17 overexpression promotes angiogenesis by increasing blood vessel sprouting and pericyte number during brain microvessel development

Juntang Lin, Cornelius Lemke, Christoph Redies, Xin Yan, Eilhard Mix, Arndt Rolfs and Jiankai Luo

Int. J. Dev. Biol. (2011) 55: 961-968

http://dx.doi.org/10.1387/ijdb.103210jl

Tryptase and chymase are angiogenic in vivo in the chorioallantoic membrane assay Domenico Ribatti, Girolamo Ranieri, Beatrice Nico, Vincenzo Benagiano and Enrico Crivellato Int. J. Dev. Biol. (2011) 55: 99-102

http://dx.doi.org/10.1387/ijdb.103138dr

Development of a fast, objective, quantitative methodology to monitor angiogenesis in the chicken chorioallantoic membrane during development

Eva Verhoelst, Bart De Ketelaere, Veerle Bruggeman, Eduardo Villamor, Eddy Decuypere and Josse De Baerdemaeker

Int. J. Dev. Biol. (2011) 55: 85-92

http://dx.doi.org/10.1387/ijdb.103119ev

5 yr ISI Impact Factor $(2011)=2.959$
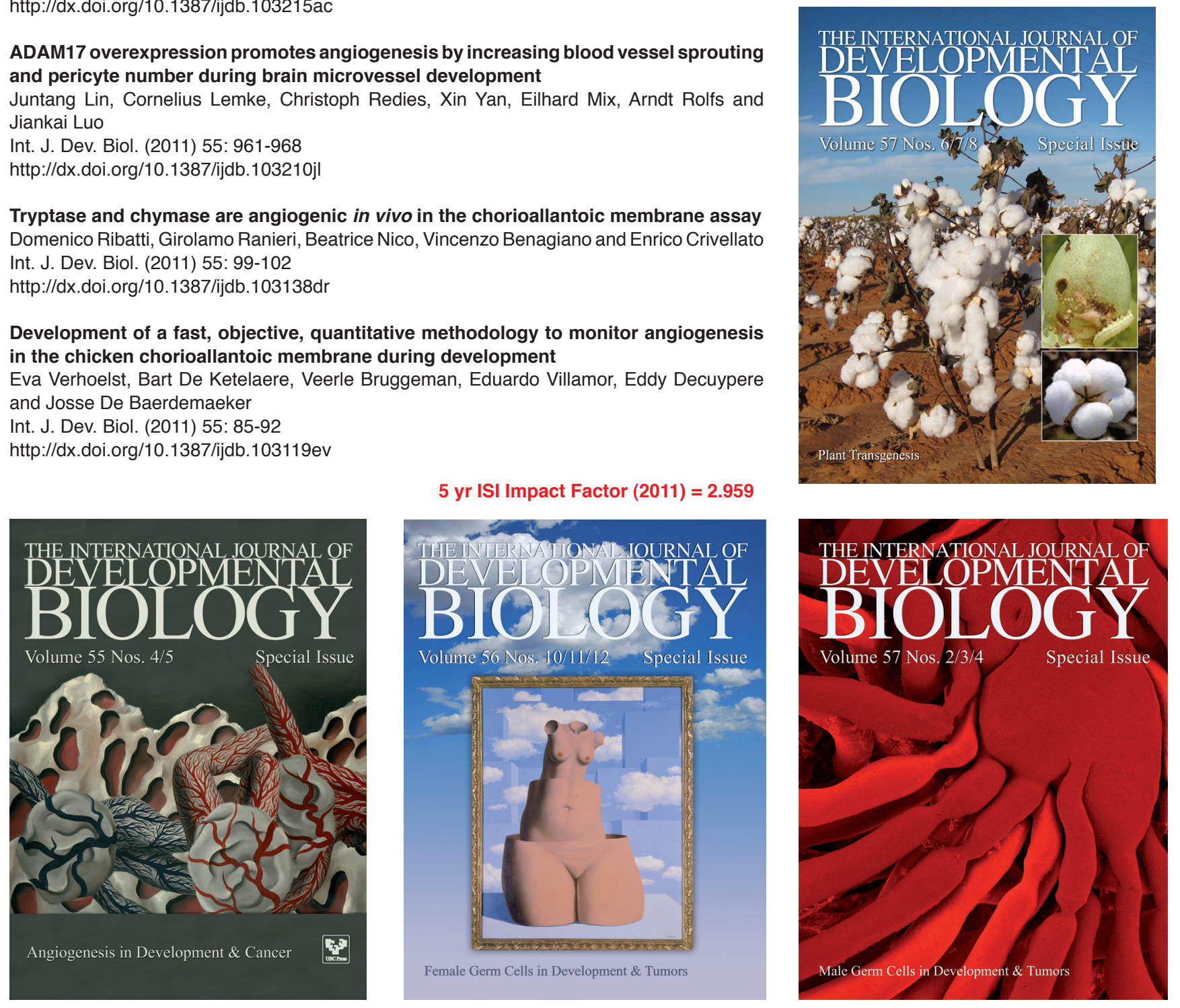